\title{
Stress Gradient Effects on Notched Composite Failure using a Local-Global Approach
}

\author{
Junghyun Ahn ${ }^{1}$, Anthony M Waas ${ }^{2}$ \\ Composite Structures Laboratory \\ Department of Aerospace Engineering, \\ University of Michigan, Ann Arbor, Ml 48109-2140
}

\begin{abstract}
Micromechanics based analysis to predict damage initiation in compressively loaded symmetric notched multidirectional laminates under remote uniaxial loading is described. A novel hybrid local-global finite element method in conjunction with the commercial code $A B A Q U S^{\circledR}$ is used to solve the governing system of equations. The results obtained for the predictions are compared against a set of experimental results. A unified model that captures damage initiation through fiber kink banding and which accounts for stress gradient effects is presented.
\end{abstract}

\section{Introduction}

Laminated fiber reinforced polymer matrix composites (PMC's) are finding increased use in a broad range of aerospace industrial applications. More importantly, PMC's are increasingly being used as primary load bearing components not just as weight saving secondary structures. These applications include one-piece fully co-cured wing structures, gas turbine composite fan blades, and fuselage reinforcement structures.

Composite materials are superior to conventional monolithic materials for a number of reasons, noteworthy amongst these being their mechanical properties. Ashby and Jones [(1994)] ${ }^{1}$ have formulated performance indices that can be used to select a material for a given application. PMC's appear to be the material of choice for several applications. Issues such as damage tolerance and durability, performance degradation due to ageing, fatigue under multiaxial loads and response at elevated temperature are currently being researched with a view to expanding the confidence levels associated with PMC applications.

Experience with previous applications of PMC's for rotor blades in the helicopter industry, pressure vessels, and other similar situations that call for superior tensile stiffness and tensile fatigue life have shown the superior performance of PMC's in a tensile environment. In contrast, compressive strength of PMC's is known to be less attractive [Mathews and Rawlings, (1994)], [Waas and Schultheisz, (1995)] $]^{3}$.

The response of composite laminates when subjected to mechanical loads is influenced by the material type (fiber and matrix) and configuration (stacking sequence, lay-up). In addition to these factors, geometrical parameters (cut-out, notch, thickness change, etc.) and loading characteristics (multiaxial, thermal, cyclic loading, etc) also affect the overall performance of composite laminates. One of the challenging tasks in the analysis and design with PMC based structural laminates is to improve predictive capability, particularly the development of suitable analyses tools to predict strength. This requires that carefully conducted experiments be carried out so that the results can be used for the development of physics based failure models that can be incorporated in finite element based numerical codes. The present paper extends the authors' previous work [Ahn and Waas, (2002)] $]^{5}$ on failure prediction in notched laminates. In particular, a micromechanics based model that consists of a region modeled discretely

\footnotetext{
${ }^{1}$ Researcher, Aerospace Engineering, University of Michigan, Ann Arbor, Copyright $(2004$ by Junghyun Ahn, published by the American Institute of Aeronautics and Astronautics, Inc; with permission

${ }^{2}$ Professor of Aerospace Engineering, Associate Fellow, AIAA.
} 
as layers of fibers and matrix material (microregion) is coupled with a homogeneous orthotropic elasticity solution for areas that are remote from the micro-region.

The development of the model relies on previous experimental work reported by the

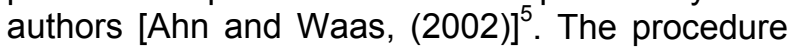
established using this model approach is found to provide an improved capability for the prediction of notched strength in multidirectional laminates.

\section{Modeling of Compressive Response of a Notched Multidirectional Laminate}

As shown in a companion paper [Ahn and Waas, (2002) $]^{5}$, when a multidirectional laminate containing a circular hole is subjected to remote compressive loads, failure in the form of a microstructural instability is initiated in the vicinity of the hole, which is the area of highest stress concentration. For cross-ply laminates, the failure is triggered by 0 -degree ply instability, characterized by fiber kinking. For the case of quasi-isotropic laminates, which consist of zero and 45-degree plies, two types of failure mechanisms compete against each other. These are failure initiation due to fiber instability in the zero plies and failure initiated by matrix shear response, responsible for interface (fiber/matrix) cracking and/or shear failure in the 45 degree plies. In the present paper, only cross-ply laminates are considered. The dominant failure mode for this type of laminates has been reported as zero ply kinking failure [Ahn and Waas (1999)] .

The micromechanics based modeling approach used in the previous paper, [Ahn and Waas (1999)] ${ }^{6}$ employed several simplifying assumptions aimed at reducing the "size" of the numerical problem. In particular, symmetry considerations were used to mesh a region that is half of the micromodel region used in the present study, in effect eliminating any non-symmetric instability modes. The overall effect of this assumption is to introduce additional stiffening, thus generating larger than measured failure initiation loads. In the present study, which is an intermediate step in the evolutionary process of developing a unified model that spans several length scales, the microregion is extended to either side of the hole as shown in Figure 1 and Figure 2. A schematic of the notched cross-ply laminates are shown in Figure 1. In Figure 1 and Figure 2, the region $A B C D$ shows the boundary of the meshed area that represents the microregion within which the fibers and matrix are modeled as discrete layers.

The failure initiation analysis based on micromechanics is carried out in a manner similar to that described in [Ahn and Waas, (1999)] $]^{6}$. For the FEA analysis, a rectangular mesh containing 16,006 elements (parabolic plane strain) and 48,533 nodes, with two degrees of freedom ( $u$ and v) per node was chosen within the microregion. Convergence of the solutions with respect to microregion size and microregion mesh density were both checked by incrementally increasing the microregion size and mesh density until no substantial change in the salient features (and values) associated with the response resulted. These features included examination of the inplane stress and displacement fields. A fully extended model (microregion embedded within a homogenized orthotropic lamina, which we refer to as the extended model) is shown in Figure 3 and is the subject of ongoing research. The Lekhnitskii orthotropic elasticity [Lekhnitskii, (1968)] ${ }^{6}$ solution for a homogenous infinite plate containing a centrally placed cutout and subjected to remote uniaxial loading is used to compute the displacement fields along the edges $B C, C D$ and DA (Figure 2) of the microregion. These displacement fields are computed corresponding to a unit far-field uniaxial compressive load.

A flow chart of the analysis procedure is indicated in Figure 4(a). First, an elastic eigenvalue analysis of the microregion model is carried out in order to obtain the eigenmodes. Next, using the eigenmode associated with the smallest non-zero eigenvalue as a perturbation to an otherwise perfect mesh, a response analysis is carried out using the arc length method option provided in $A B A Q U S^{\circledR}$. During the response analysis, the boundaries $B C, C D$ and $D A$ are subjected to displacement fields that are computed from the orthotropic elasticity solution and the edge $A B$ is subjected to the equilibrium traction fields computed from the same solution and corresponding to the uniform pre-kinking state. As shown previously in Ahn and Waas $(2002)^{5}$, the maximum load prediction is insensitive to the details of the pre-kinking boundary tractions on edge $A B$ and in the work reported here, this edge was kept free during the response analyses. Simulations were carried out for a series of imperfection magnitudes for each hole size. The eigenmodes provide the perturbation shape for the initial fiber misalignment but not the absolute magnitude of perturbation. Thus, the imperfection magnitude must be 
specified by the user. In the present work, this is achieved as follows; as shown in Figure 4(b), the maximum amplitude of the lowest eigenmode (which occurs at the free notch edge - indicated as point $J$ in figure 2) is chosen such that the fiber misalignment angle $\phi$ can be controlled. Since $\lambda$ is known, $\delta$ is chosen such that $\phi$ assumes the intended value. Several simulations corresponding to different values of $\phi$ were completed. In each case, the maximum load associated with the global load proportionality factor applied in proportion to the initial displacement field is computed. In a typical run, the relation between the applied load and the axial displacement (average axial displacement of the microregion along the edge $B C$ ) is as shown in Figure 4(a) inset "response of microregion". The load rises almost linearly, followed by a well-defined peak load and subsequent snap back in the response. The peak load corresponds to the onset of instability while the snap back part of the response corresponds to the formation of a kink band (in which the deformation is localized) with simultaneous growth and rotation of the kink band. The far-field applied loads corresponding to the peak loads can be plotted as a function of imperfection magnitude, see Figure 4(a) - inset "extrapolation to perfect case", from which, by extrapolation, the maximum applied stress corresponding to perfectly straight fibers can be extracted. Thus, the process outlined not only gives the maximum strength of the laminate corresponding to the given (or measured) fiber misalignment, but also provides the maximum attainable strength of the laminate. The latter could be used as an upper bound on strength, while the strength corresponding to a fiber misalignment of $1.5^{\circ}$ (say) could be used as the lower bound.

Throughout the analyses, the fiber (IM7) is assumed to be linearly elastic (see Table 1), and the matrix (977-3 toughened epoxy) property within the laminate (in-situ) is evaluated from a $+45 /-45$ laminated coupon test, which will be described in the next section. Thus, the in-situ elastic plastic matrix behavior is incorporated in the present analysis. The matrix is modeled as a J2 incremental flow theory solid with isotropic hardening, and this is also verified through measurement.

\section{In-Situ Matrix Characterization}

To evaluate the material properties of the matrix within the laminate (in-situ matrix properties), a $( \pm 45)_{n s}$ coupon test (Figure 5) at room temperature was performed following the procedure of ASTM-D3518-76 [ASTM Standard, $(1982)]^{8}$. The elastic properties of the lamina and the complete shear stress-shear strain behavior of an IM7/977-3 in the principal material coordinate system were obtained from this test. The procedure used to generate the data consists of subjecting $\mathrm{a}( \pm 45)_{n s}$ angle ply laminate to uniaxial compression and measuring the laminate strains $\varepsilon_{x x}$ and $\varepsilon_{y y}$ and the applied remote stress on the laminate. Note that, for this test, $\gamma_{x y}=\varepsilon_{x x}-\varepsilon_{y y}$. The data shown in Figure 6 from this test can be used to extract the complete nonlinear shear stress shear strain response of the in-situ matrix (977-3) as discussed below. We begin by assuming that the 977-3 matrix material can be modeled as an elastic-plastic solid obeying the small strain J2 flow theory of plasticity [Lubliner, (1998)] $]^{9}$. Then, from the elastic (linear) portion of the curve in Figure 6, we first obtain the inplane lamina shear modulus $G_{12}$. In the lamina principal coordinates, we know that,

$$
\begin{aligned}
& \varepsilon_{11}=\varepsilon_{22}=\frac{\varepsilon_{x x}+\varepsilon_{y y}}{2} \\
& \gamma_{12}=2 \varepsilon_{12}=\varepsilon_{x x}-\varepsilon_{y y}
\end{aligned}
$$

And

$$
\begin{aligned}
& \sigma_{11}=\frac{\sigma_{x x}}{2} \\
& \sigma_{22}=\frac{\sigma_{x x}}{2} \\
& \tau_{12}=\frac{\sigma_{x x}}{2}
\end{aligned}
$$

Thus, using (1) and (2) above and the definitions of equivalent stress, $\bar{\sigma}$ and equivalent plastic strain increment, $d \bar{\varepsilon}^{p}$ [Lubliner, (1998)] , the data in Figure 7 can be used to construct a plot of $\bar{\sigma}$ against $\bar{\varepsilon}^{p}$.

According to the $\mathrm{J} 2$ flow theory of plasticity with a Mises-Henky yield condition, the ratio of the increment of each plastic strain component to its corresponding deviatoric stress component remains constant, which is [Lubliner, (1998)]",

$$
\frac{d \varepsilon_{i j}^{p}}{\hat{\sigma}_{i j}}=d \lambda
$$

Using (3), and the relation between $\bar{\sigma}$ and $d \bar{\varepsilon}^{p}$, the nonlinear portion of the shear stress $\left(\tau_{12}\right)$ - 
shear strain $\left(\gamma_{12}\right)$ response curve for a single lamina can be extracted. The curve thus obtained for IM7/977-3 system is shown in Figure 8. The instantaneous slope of the curve in Figure 8 is the tangent shear modulus of the lamina, $G_{12}^{T}\left(\tau_{12}\right)$, while the ratio of shear stress to shear strain is the secant modulus. The approximate Halpin-Tsai relations [Daniel and Ishai, (1994)] ${ }^{10}$ are used to extract the variation of the in-situ matrix shear modulus $G_{m}^{S}\left(\tau_{12}\right)$,

$$
\begin{aligned}
G_{m}^{S} & =G_{12}^{S}\left(\frac{1+\xi_{2} \cdot \eta_{2} \cdot v_{f}}{1-\eta_{2} \cdot v_{f}}\right)^{-1} \\
\eta_{2} & =\frac{G_{12 f}-G_{m}}{G_{12 f}+\xi_{2} G_{m}}
\end{aligned}
$$

and $\eta_{2}=1$ for random packing of fibers. With $G_{m}^{S}\left(\tau_{12}\right)$ so obtained, the in-situ matrix shear stressshear strain curve is as shown in Figure 9. In a similar manner, the in-situ uniaxial stress-strain curve in compression for the matrix is also obtained (Figure 10). This uniaxial response curve was discretized and used in the input data file for the micromechanics based finite element analysis carried out using ABAQUS ${ }^{\circ}$.

\section{FEA Results and Interpretation}

A typical load response behavior of a microregion within a cross ply laminate model is shown in Figure 11 and a series of deformed plots of the microregion showing the initiation and propagation of damage in the form of kink banding is as shown in figure 12, for a specimen with hole size 0.25 inch radius. The numbers indicated in the plot of Figure 11 correspond to the series of deformation plots shown in Figure 12.

The microregion response follows a linear path up to point (1). Although local matrix yielding (in areas of the microregion near the cutout) is indicated prior to the attainment of point(2), the total integrity of this region is not affected much from the matrix yielding because, (1) the area of yielding is small compared to the overall size of the microregion and (2) the fiber rotations are small up to the point of maximum load (point(3). Therefore, the reaction force averaged along the edge $\mathrm{BC}\left(\mathrm{RF}_{1}\right)$ vs. far field load relation is linear up to the maximum load point. As loading increases, the fibers in the areas where the matrix has become "softer" start to rotate, resulting in a drop of the resultant force. Once the load drop initiates (characterized by high localized deformation at the hole center), the band of localized deformation continues to propagate into the unkinked material until it reaches the outer micromodel boundary. Simulations corresponding to several different fiber misalignment angles were carried out and the results for maximum load so obtained were plotted against imperfection amplitude in order to determine the upper bound strength corresponding to perfectly straight fibers.

Another failure mechanism observed with laminates that include a dominant number of offaxis plies was matrix shearing eventually leading to fiber/matrix interface fracture. An analysis that includes incorporation of cohesive zone models much in the spirit of [Song and Waas (1995)] ${ }^{11}$, is relegated to the future. For now, we observe the good agreement between the far-field load corresponding to the maximum load prediction and the experimentally measured failure loads for kink banding as indicated in Table 2, especially for the case of small imperfection $\left(\phi=1.5^{\circ}\right)$ and a hole size of 0.25 inch radius. Table 2 also provides values for the upper and lower bound compressive strength as a function of hole size. The results in Table 2 indicate notch sensitivity. The trends for compressive strength as a function of hole radius are very similar to that reported experimentally for comparable fiber/matrix systems (for instance see Soutis et al. (1991) $)^{12}$ and Starnes and Williams $(1982)^{13}$. The good agreement between prediction and experiment for the single hole size that we examined experimentally lends confidence to our contention that zero ply kinking is the dominant and hence governing mode of failure initiation in notched cross-ply laminates. Such a conclusion, based only on experimental results, has been reached earlier by several previous investigatorssee for example, Soutis, Fleck and Smith $(1991)^{12}$ and Khamseh and Waas (1997) ${ }^{14}$.

\section{Concluding Remarks}

A micromechanics based finite element localglobal model for predicting the compressive strength of notched cross-ply composite laminates has been presented. The effect of notch size (and hence the stress gradients that are generated by the notch) has been adequately captured and results in notch sensitivity as has been observed experimentally. The very good comparison between experiment and analysis for a single notch size lends confidence to the veracity of the model. Using measured fiber and matrix nonlinear material properties, geometry and stacking of the laminate, the model has accurately predicted the 
notched compressive strength of laminates and the sensitivity of strength to notch size.

\section{Acknowledgments}

We are grateful for the partial support from the AFOSR and the University of Michigan for this work. This paper is based, in part on the Ph.D. thesis of the first author submitted and defended in the Aerospace Engineering Department, University of Michigan, in December 1999.

In addition, the authors appreciate assistance of Moonjung Kim and the support of the 2003 MIT/UTC Pratt \& Whitney SDM group members.

\section{References}

1. Ashby M.F. and Jones, D., "Engineering Materials 2", Pergamon, 1994

2. Mathews, F. L. and Rawlings, R., "Composite Materials: Engineering and Science", Chapman and Hall, London, 1994

3. Waas, A. M., and Schultheisz, C. R., "Compressive failure in Composites, Part II", Progress in Aerospace Science, Vol.32, pg. 43 - 78, 1995.

4. Starnes, J., Rhodes, M.D., and Williams, J.G., "Effect of Impact Damage and Holes on the Compressive Strength of a Graphite/Epoxy Laminate", Nondestructive Evaluation and Flaw Criticality for Composite Materials, edited by R. B. Pipes, ASTM STP 696, pg. 145-171, 1979.

5. Ahn, Junghyun and Waas, A.M., "Prediction of Compressive Failure in Laminated Composites at Room and Elevated Temperature", AIAA JOURNAL, Volume 30, Number 2, pg. 346 - 368, 2002

6. Ahn, Junghyun and Waas, A.M. "Finite Element Model for Compressive Failure of Notched Uniply Composite Laminates under Remote Biaxial Loads" ASME Journal of Engineering Materials and Technology: Vol.121, pg. $001-007,1999$

7. Lekhnitskii S., "Theory of Elasticity of an Anisotropic body", Text, Godden-Day, 1968.

8. "D 3410-75 Standard Test Method for Compressive Properties of Unidirectional or Cross-ply Fiber-Resin Composites", 1982, Annual Book of ASTM Standards, Part 36, American Society for Testing and Materials, Philadelphia, PA, pg. 872-880., 1982

9. Lubliner Jacob, "Plasticity Theory", Text, Prentice Hall, 1998.
10. Daniel, Isaac M. and Ori Ishai, "Engineering Mechanics of Composite Materials", Oxford University Press, New York, 1994.

11. Song, S.J. and Waas, A.M., "An Energy Method based Model for Mixed Model Failure of Laminated Composites", AIAA Journal, vol. 33, No. 4, pg. 739-745, 1995.

12. Soutis, C., Fleck, N. and Smith, F., "Failure Prediction Technique for Compression Loaded Carbon Fiber-Epoxy Laminate with an Open Hole", J. Composite Materials, 25, pg. 14761498, 1991

13. Starnes, J. and Williams, J.G. (1982), Failure characteristics of Graphite/Epoxy structural components loaded in compression, NASA TM 84552.

14. Khamseh A. and Waas A., "Failure Mechanisms of Composite Plates with a Circular Hole under remote Biaxial Planar Compressive Loads", ASME J. Materials and Technology, vol. 119, pg. 56-64, 1997 


\section{Tables and Figures}

\begin{tabular}{|c|c|c|c|c|c|}
\hline Material & $\begin{array}{c}\mathrm{E}_{11} \\
(\mathrm{Msi})\end{array}$ & $\begin{array}{c}\mathrm{E}_{22} \\
(\mathrm{Msi})\end{array}$ & $\begin{array}{c}\mathrm{G}_{12} \\
(\mathrm{Msi})\end{array}$ & $v_{12}$ & $\begin{array}{c}\text { Thickne } \\
\text { ss (in) }\end{array}$ \\
\hline $\begin{array}{c}\text { IM7/977- } \\
\text { 3 Epoxy }\end{array}$ & 23.5 & 1.21 & 0.72 & 0.30 & 0.0052 \\
\hline IM7 Fiber & 42 & & & 0.25 & $\begin{array}{c}2.756 \\
\mathrm{E}-04\end{array}$ \\
\hline $\begin{array}{c}977-3 \\
\text { Epoxy }\end{array}$ & 0.7 & & & 0.34 & $\begin{array}{c}2.444 \\
\mathrm{E}-04\end{array}$ \\
\hline
\end{tabular}

Table 1 Zero ply material properties of the $\mathbf{4 8}$ ply graphite / 977- 3 epoxy composites

\begin{tabular}{|c|c|c|c|}
\hline Hole Radius (in) & 0.0625 & 0.125 & 0.25 \\
\hline $\begin{array}{c}\text { Max. Load (no } \\
\text { imperfection) } \\
\text { (ksi) }\end{array}$ & 163 & 123 & 75 \\
\hline $\begin{array}{c}\text { Max. Load (1.5 deg } \\
\text { imperfection) } \\
\text { (ksi) }\end{array}$ & 78 & 63 & $\begin{array}{c}44 \\
\text { (matching } \\
\text { test result) }\end{array}$ \\
\hline
\end{tabular}

Table 2 Analyses Results

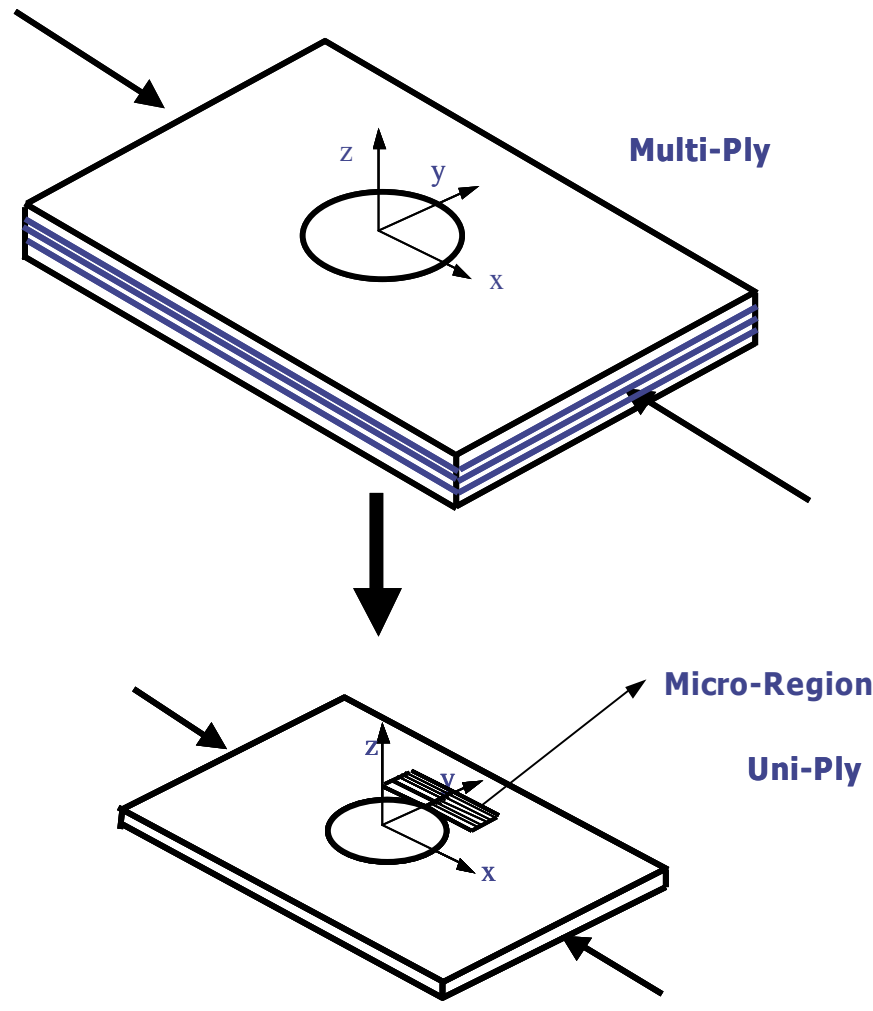

Figure 1. Problem configuration for Crossply Laminates

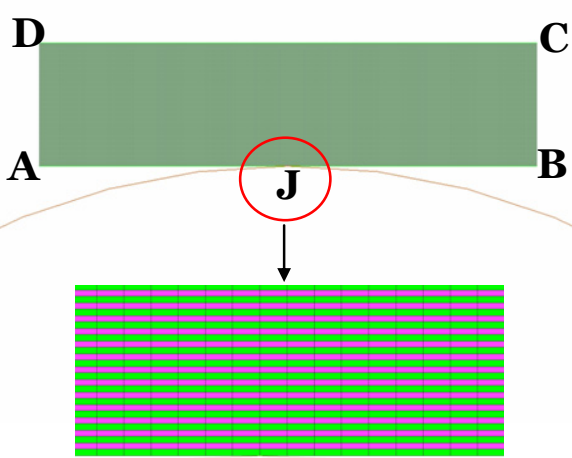

Figure 2. Full Model

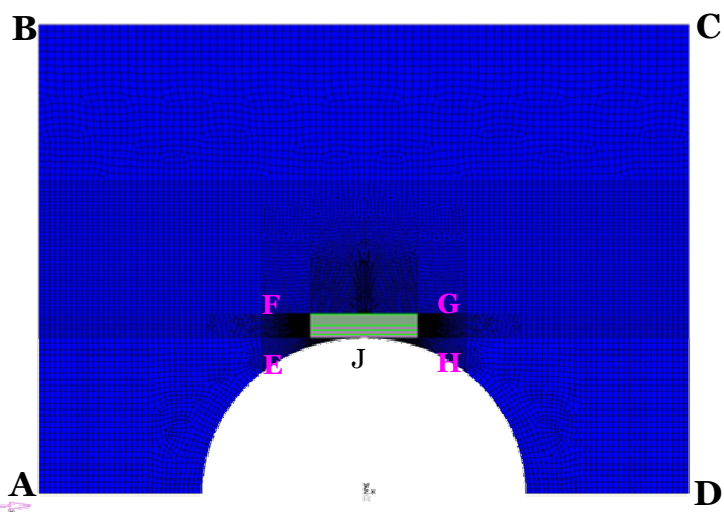

Figure 3. Extended Model

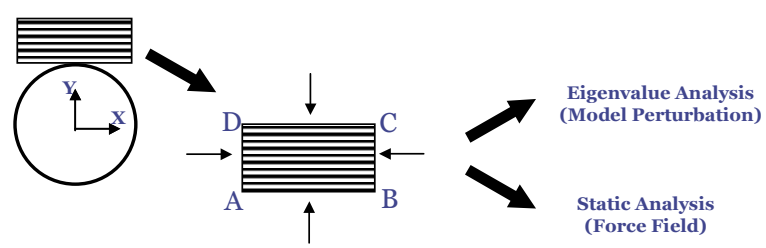

Nonlinear Response Analysis

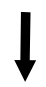

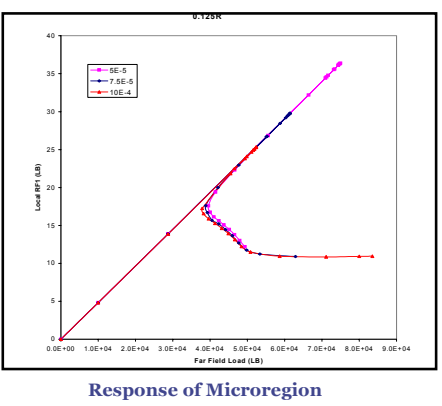

Response of Microregion

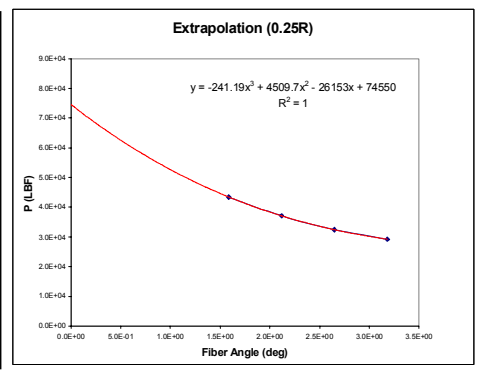

Extrapolation to Perfect Case
Figure 4(a) Imperfection Sensitivity Analysis 
AIAA-2004-1844

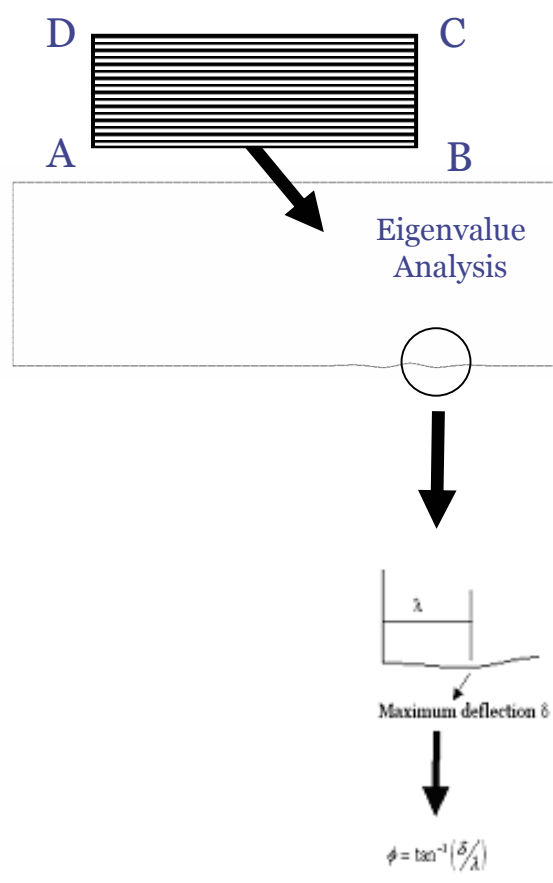

Figure 4(b) Details of initial imperfection



Figure 5. $+45 /-45$ coupon test of a specimen taken from the cross ply laminates 
AIAA-2004-1844
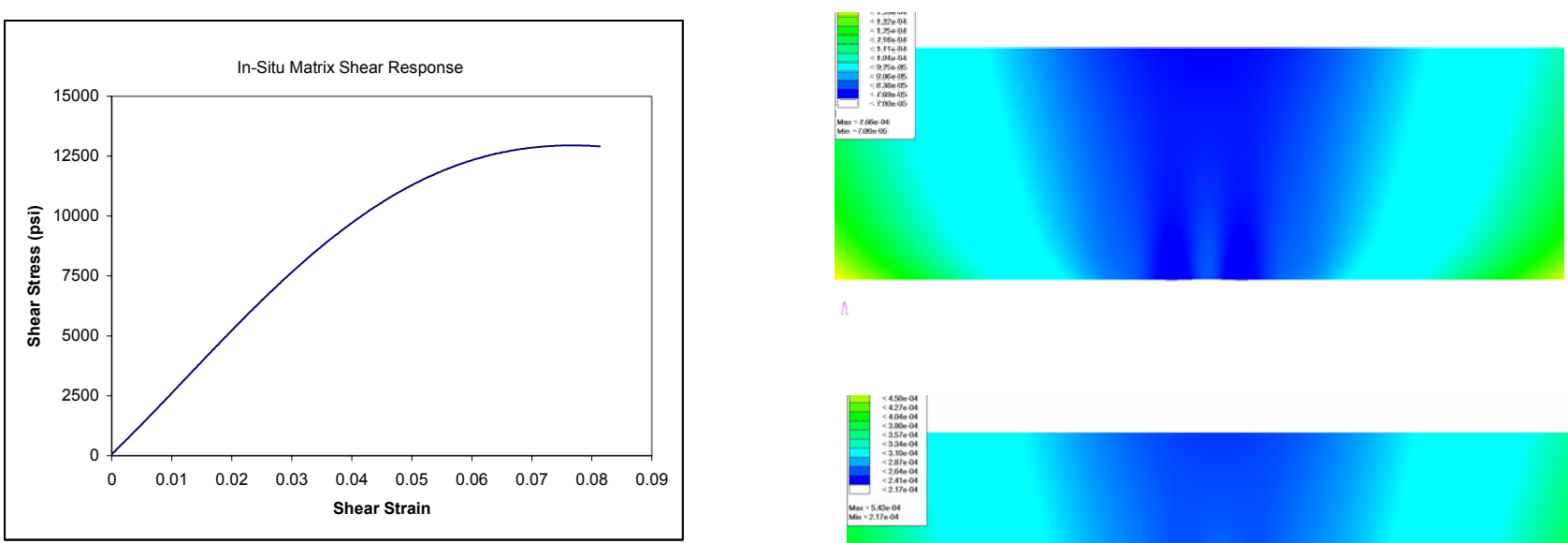

Figure 9. The in-situ Shear Stress vs. Shear Strain response of the matrix.
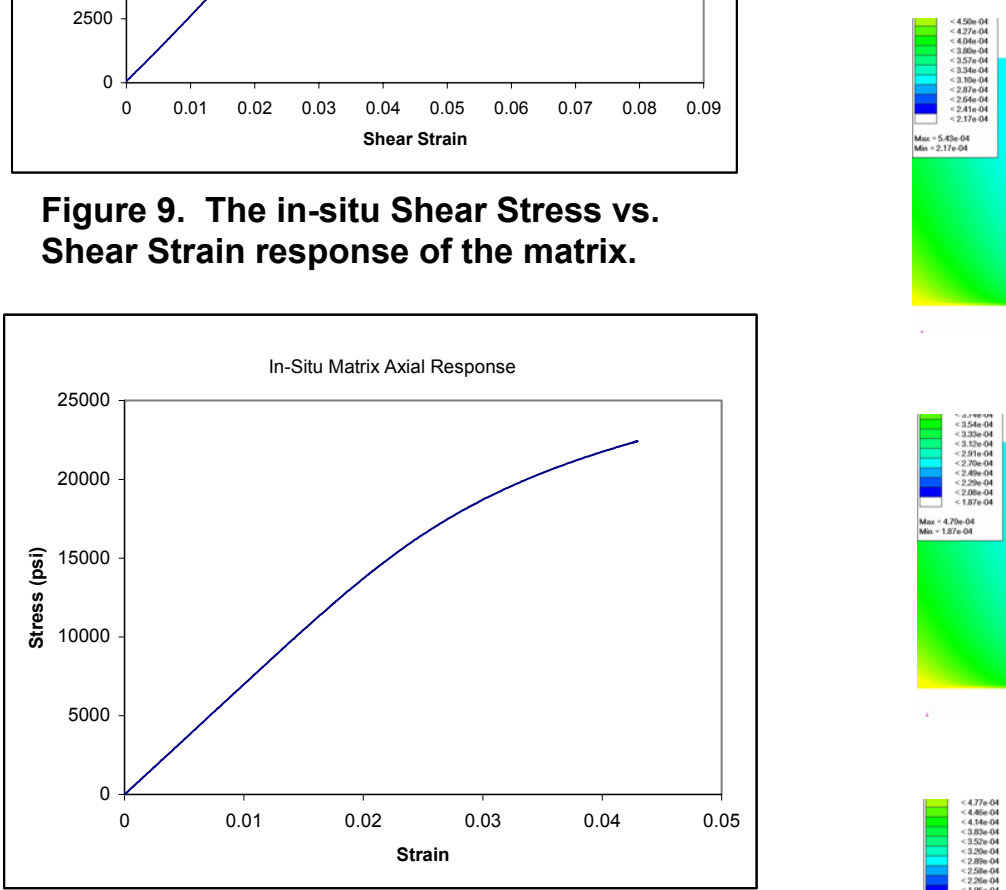

Figure 10. The in-situ uniaxial stress vs. uniaxial strain response of the matrix.
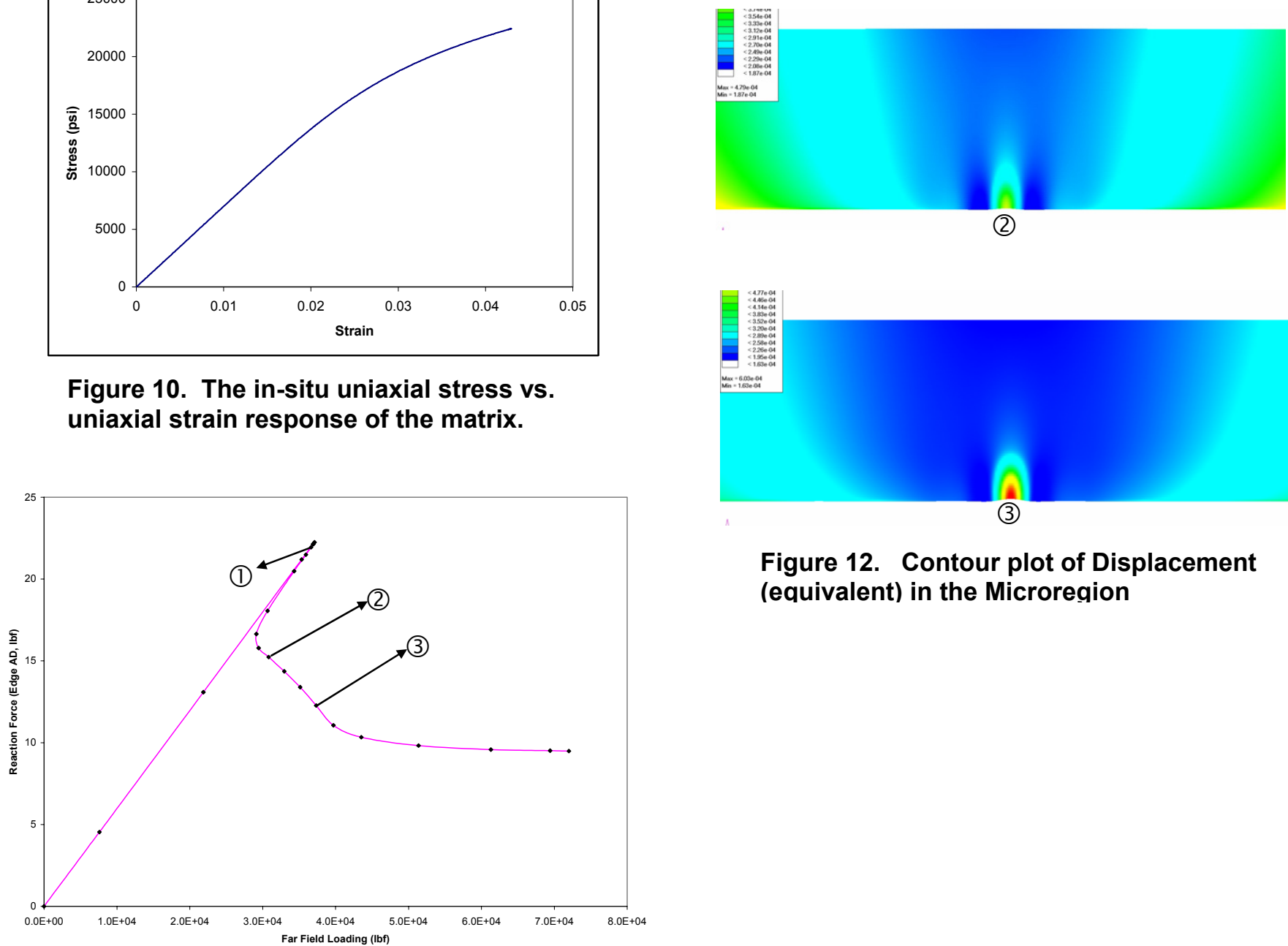

(2)

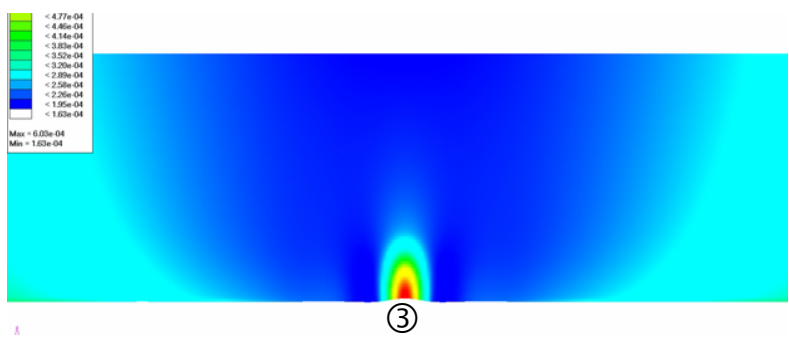

Figure 12. Contour plot of Displacement (equivalent) in the Microregion

Figure 11. Typical Response Curve of the microregion $(0.25 R)$ 